\title{
Pipel: exploiting resource reorganisation to optimise performance of pipeline-structured applications in the cloud
}

\section{Vinicius Meyer, Vinicius Facco Rodrigues, Rodrigo da Rosa Righi* and Cristiano André da Costa}

Applied Computing Graduate Program, Unisinos University, Av. Unisinos, 950 - Cristo Rei, São Leopoldo, Rio Grande do Sul, Brazil

Email: vinimeyer1@gmail.com

Email: vfrodrigues@unisinos.br

Email: rrrighi@unisinos.br

Email: cac@unisinos.br

*Corresponding author

\section{Guilherme Galante}

Western Paraná State University - UNIOESTE,

R. Universitária, 2069 - Jardim Universitário,

Cascavel, Paraná, Brazil

Email: gui.galante@gmail.com

\section{Cristiano Bonato Both}

Federal University of Health Sciences of Porto Alegre - UFCSPA,

R. Sarmento Leite, 245 - Centro Histórico,

Porto Alegre, Rio Grande do Sul, Brazil

Email: cbboth@inf.ufrgs.br

\begin{abstract}
Workflow has become a standard for many scientific applications that are characterized by a collection of processing elements. Particularly, a pipeline application is a type of workflow that receives a set of tasks, which must pass through all processing elements in a linear fashion. However, the strategy of using a fixed number of resources can cause under- or over-provisioning situations, besides not fitting irregular demands. In this context, our idea is to deploy the pipeline application in the cloud, so executing it with a feature that differentiates cloud from other distributed systems: resource elasticity. Thus, we propose Pipel: a reactive elasticity model that uses lower and upper load thresholds and the CPU metric to on-the-fly select the most appropriated number of compute nodes for each stage along the pipeline execution. The results were promising, presenting an average gain of $38 \%$ in the application time when comparing non-elastic and elastic executions.
\end{abstract}

Keywords: cloud elasticity; pipeline applications; performance optimisation; dynamic resource management; adaptivity.

Reference to this paper should be made as follows: Meyer, V., Rodrigues, V.F., Righi, R.d.R., da Costa, C.A., Galante, G. and Both, C.B. (2019) 'Pipel: exploiting resource reorganisation to optimise performance of pipeline-structured applications in the cloud', Int. J. Computational Systems Engineering, Vol. 5, No. 1, pp.1-17.

Biographical notes: Vinicius Meyer received his Bachelor's degree in Computer Engineering at the Univates in 2014 and his Master's degree in Applied Computing at the Unisinos University in 2016. His research areas include computer networks and distributed systems. Currently, he is researching elasticity for parallel applications in cloud computing environment.

Vinicius Facco Rodrigues received his Master's degree in Applied Computing in 2016 at Unisinos. Currently, he is a PhD student in Applied Computing and researcher at the same university. His research areas include distributed systems and computer networks. Currently, he is focusing his research on the topic of cloud computing and, more specifically, on the elasticity feature of this new paradigm. 
Rodrigo da Rosa Righi is an Assistant Professor and researcher at Unisinos University. He concluded his post-doctoral studies at KAIST - Korea Advanced Institute of Science and Technology, under the following topics: RFID and cloud computing. He obtained his PhD in Computer Science from the UFRGS University, Brazil, in 2005. His research interests include load balancing and process migration. Finally, he is a member of the IEEE and ACM.

Cristiano André da Costa is a Full Professor at Unisinos and a researcher on productivity at $\mathrm{CNPq}$ (National Council for Scientific and Technological Development). His research interests include ubiquitous, mobile, parallel and distributed computing. He obtained his $\mathrm{PhD}$ degree in Computer Science from the UFRGS University, Brazil, in 2008. He is a member of the ACM, IEEE, IADIS and the Brazilian Computer Society (SBC).

Guilherme Galante is a Professor at Western Paraná State University. He received his $\mathrm{PhD}$ at UFRGS in 2014. His research is focused on applied computing, cloud and high performance computing.

Cristiano Bonato Both is an Associate Professor at the Federal University of Health Sciences of Porto Alegre (UFCSPA), Brazil. He holds a Postdoctoral title in Computer Science at the Institute of Informatics of the Federal University of Rio Grande do Sul (UFRGS), Brazil. He received his $\mathrm{PhD}$ degree at UFRGS in 2011 and his MSc in Computer Science from the Ponthifical Catholic University of Rio Grande do Sul, Brazil, in 2003. His research interests include wireless networks, next generation networks, and software-defined networking.

\section{Introduction}

Workflow computing has become a main stream to model many scientific applications (Zhang et al., 2015; Wu et al., 2016; Hendrix et al., 2016). This kind of demand are normally represented by direct acyclic graphs (DAGs), where there are processing elements and an arbitrary exchange of data among them. On such programming model, we dispose of a collection of input tasks that must be processed along the workflow, so the application performance is normally measured as either the final time to compute a finite number of tasks or system throughput. The DAG organisation is very dependent of the application, but there is a particular type of workflow that are receiving more and more focus thanks to being also a parallel programming model (Ramanath et al., 2005), the pipeline. Here, the processing elements, denoted as stages, are organised in a linear way, where the output of one stage is the input of the next one. This type of application is generally applied in

1 image processing, where the flows of images enter the pipeline and pass through various steps, such as filters, encoders, and so on (Ramanath et al., 2005; Guirado et al., 2006; Hartley et al., 2009)

2 video processing (Schneider et al., 2009).

Pipeline-structured applications can easily become time-consuming, since performance depends on the number of input tasks, features of each task, the number of stages and the processing behaviour of each stage (Rodriguez and Buyya, 2015; Jahn et al., 2015).

A traditional problem in pipeline applications occurs when a stage becomes slower than others, so being the system bottleneck (Malawski et al., 2012; Beltran, 2015). The insertion of bubbles along the stages or the presence of large buffers in front of each stage acts as palliative strategies to bypass such trouble. In this case, it is possible to explore the idea of superscalar pipelines as an alternative to enhance system throughput (Mattoso et al., 2010; Costa et al., 2012). In superscalar pipelines, the processing power of each stage is replicated, so we can have two or more tasks being processed at each stage concomitantly. Thus, unlike using a single compute node to address a particular stage, it is possible to distribute its incoming tasks among the nodes of a cluster. However, the strategy of using a fixed number of resources can cause under-or over-provisioning situations, besides not fitting irregular demands (Rodriguez and Buyya, 2015). In addition, selecting the number of resources and their configurations are not trivial procedures, being strongly dependent of the application and the tasks to be processed.

In addition to clusters, we can observe the emergence of cloud computing addressing scalability and powerful resources, both required by high performance computing (HPC) applications. Cloud computing has a feature that is not found on traditional distributed systems: resource elasticity (Righi et al., 2015). Elasticity can be defined as the ability of a system to dynamically add or remove computational resources used by an application to match the current demand as closely as possible (Galante and de Bona, 2012). Thus, elasticity can be used to find the most appropriate set of resources to run pipeline applications whose requirements like the number of stages and input tasks, as well as the features of stages and tasks, sometimes cannot be determined in advance. In the best of our knowledge, the current state-of-the-art on cloud elasticity does not contemplate pipeline applications looking at the resource needs of each stage, being mainly explored for Web applications (Beltran, 2015; Sharma et al., 2011) or master-slave HPC demands (Rajan et al., 2011; Righi et al., 2015).

Aiming at exploring the benefits of elasticity to pipeline applications, we are proposing a model named Pipel. Pipel provides an architecture in which we analyse horizontal elasticity in the scope of each stage, which has a controller that manages load balancing and scaling in and out 
operations [i.e., virtual machine (VM) consolidation and allocation]. Acting at the platform as a service (PaaS) level of a cloud, Pipel is capable of transforming a non-elastic pipeline application in an elastic one without any intervention of the programmer. The main advantage on using a malleable number of resources at each stage is to provide the same cadence for the whole system, so when a task leaves a stage the next one is fully ready to receive and process it.

This article presents the Pipel architecture, highlighting load balancing and equations and rules for scaling in and out operations. Considering the Pipel contributions, they are classified as twofold:

1 an architecture that provides elasticity for pipeline applications where the control grain for resource reorganisation refers to each stage of the application

2 we introduce the concept of asynchronous multielasticity, which enables the elasticity controllers at each stage to perform scaling in and out operations without blocking the normal execution of the application.

Based on Pipel, we developed a prototype which was evaluated with a three stages graphical application which was observed against two scenarios: elastic and non-elastic. Both scenarios were tested with four different workloads:

1 increasing

2 decreasing

3 constant

4 oscillating.

The use of synthetic workloads is considered a representative form to evaluate elasticity in computational clouds (Islam et al., 2012). The results are promising, presenting an average gain of $38 \%$ in the application time when comparing non-elastic and elastic executions.

The remainder of this article will first introduce related work in Section 2. Section 3 describes the Pipel model, presenting its architecture and components. Sections 4 and 5 present the evaluation methodology and the discussion of the results, respectively. Finally, Section 6 shows the concluding remarks, emphasising the Pipel contributions and suggesting future works for the next steps of this research.

\section{Related work}

This section presents research initiatives related to the execution of either pipeline applications or HP demands in the cloud. Table 1 presents the selected articles, which are classified in accordance with five criteria that we considered as relevant. The criteria are explained below:

- cloud level: refers to the operation level in which the model is applied (SaaS, IaaS or PaaS)

- $\quad$ elasticity method: refers to the elasticity method employed (horizontal or vertical)

- elasticity model: refers to the model employed to trigger the elasticity actions

- elasticity by stage: if a pipeline or workflow application, does it apply elasticity by stage

- application model: what are the application models that the proposed solution supports

- $\quad$ workload: for what kind of workload the model was designed.

Table 1 Related work comparison

\begin{tabular}{|c|c|c|c|c|c|c|}
\hline Research & $\begin{array}{l}\text { Cloud } \\
\text { level }\end{array}$ & Elasticity method & Elasticity model & $\begin{array}{l}\text { Elasticity by } \\
\text { stage }\end{array}$ & Applic. model & Workload \\
\hline Li et al. (2010) & PaaS & Horizontal & Manual & Not available & Pipeline & Satellite image reduction \\
\hline Rajan et al. (2011) & PaaS & Horizontal & Manual & Not applicable & Master slave & $\begin{array}{c}\text { Dynamic molecular } \\
\text { exchange }\end{array}$ \\
\hline $\begin{array}{l}\text { Mao and } \\
\text { Humphrey (2011) }\end{array}$ & IaaS & Horizontal & $\begin{array}{c}\text { Automatic, reactive and } \\
\text { proactive }\end{array}$ & Not available & Pipeline & $\begin{array}{l}\text { Several workflow } \\
\text { applications }\end{array}$ \\
\hline $\begin{array}{l}\text { Sharma et al. } \\
(2011)\end{array}$ & IaaS & Horizontal & $\begin{array}{c}\text { Automatic, reactive and } \\
\text { proactive }\end{array}$ & Not applicable & Bag of tasks & E-commerce \\
\hline $\begin{array}{l}\text { Apostol et al. } \\
(2011)\end{array}$ & IaaS & Horizontal & $\begin{array}{c}\text { Automatic, reactive and } \\
\text { proactive }\end{array}$ & Not applicable & Not informed & Not informed \\
\hline Imai et al. (2012) & PaaS & Horizontal & Automatic and proactive & Not applicable & Not informed & Heat diffusion \\
\hline $\begin{array}{l}\text { Zhang et al. } \\
(2012)\end{array}$ & IaaS & Horizontal & Automatic and proactive & Not available & $\begin{array}{c}\text { Workflow and } \\
\text { bag of tasks }\end{array}$ & Commercial application \\
\hline $\begin{array}{l}\text { Rodriguez and } \\
\text { Buyya (2015) }\end{array}$ & IaaS & Horizontal & $\begin{array}{c}\text { Automatic, reactive and } \\
\text { proactive }\end{array}$ & Not available & $\begin{array}{l}\text { Pipeline and } \\
\text { bag of tasks }\end{array}$ & $\begin{array}{l}\text { Several workflow } \\
\text { applications }\end{array}$ \\
\hline Righi et al. (2015) & PaaS & Horizontal & Automatic and reactive & Not applicable & Master slave & $\begin{array}{c}\text { Differential equations } \\
\text { calculation }\end{array}$ \\
\hline Beltran (2015) & PaaS & Horizontal vertical & Automatic and reactive & Not available & Pipeline & Multi-tier applications \\
\hline Jahn et al. (2015) & IaaS & Horizontal & Manual & Not available & Pipeline & Pipeline applications \\
\hline
\end{tabular}


Li et al. (2010) presents a model to reduce the processing time of data obtained from satellites. To accomplish this, the authors use an application in a cloud-based pipeline format, so aiming at hiding the complexity of processing and transformation of data to the end users. This pipeline is flexible and extensible to accommodate different scientific data processing tasks, and can be scaled dynamically to meet different computational requirements. Rajan et al. (2011) studied the disadvantages of parallel computing as a platform to run high-performance scientific applications. In this work, the authors describe an elastic deployment behaviour on different platforms, including Amazon EC2 and Windows Azure. They execute a molecular dynamic exchange application that explores the master-slave parallel computing model.

Mao and Humphrey (2011) developed a mechanism of cloud computing resource scaling for workflow applications. The goal is to finish all the work within the time specified by the user more efficiently and consuming the least possible amount of resources. The tests were performed on three types of workflows: Pipeline, parallel and hybrid. This approach was evaluated in four workload standards (stable, growing, oscillatory and interleaved in low and high), showing a reduction in cost from $9.8 \%$ to $40.4 \%$, when compared to other approaches. In order to improve performance, also reducing the costs in a cloud environment, Sharma et al. (2011) have created a prototype called KingFisher. Through a cloud platform provider, this model provides support for implementation and management of VM images in conjunction with elasticity feature. In particular, by using the XML-RPC API they create, consolidate or reconfigure VM.

In 2011, Apostol et al. present a management mechanism for cloud computing systems. The proposed model allocates virtual resources flexibly, taking into account the time, the cost and physical resources. It provides elasticity on demand or based on predefined policies. Through a genetic algorithm, the authors evaluate the 'quality' (resources) of populations (VMs) according to their characteristics (CPU, RAM and disk). Imai et al. (2012) proposed cloud operation system (COS). It is a middleware to support workloads providing elasticity without user intervention in the cloud. The work is based on a VM reconfiguration implementation strategy to improve performance and reducing energy consumption. To scale a workload the model creates new VMs and migrates part of the application to them. When a node gets idle, the middleware also can turn it off. Through an automatic and proactive elasticity, COS resource manager decides to create a new VM only if it received high CPU utilisation events.

Zhang et al. (2012) developed an algorithm for computational cloud environments that have the function of balancing the resources (VMs) in order to reduce the risk of overloading the cloud data centre. This algorithm considers CPU load of the VMs in addition to network communication among them. First the algorithm performs an analysis of the entire cloud, then searches the best VM to be migrated and after that looks for the best node to receive this VM. Rodriguez and Buyya (2015) present an approach to study computational cloud scaling of resources for scientific workflow applications. The authors explored the IaaS service model with the allocation of tasks on VMs, in order to use the minimum necessary of VMs to run the application as efficient as possible. In the simulation process, it was modelled a data centre with four types of VMs and elastic resources were applied on demand. This model exploits the resource provisioning strategy that works in conjunction with an scheduling algorithm. It is also used a heuristic to decide not only the type and number of VMs, but also when is the best time to allocate and deallocate them.

Righi et al. (2015) proposed a model for elastic HPC applications based on PaaS level of cloud entitled AutoElastic. In this approach, only master-slave applications are supported, exploring different types of binaries in the multiple program multiple data (MPMD) style. The authors explored horizontal and reactive elasticity using the CPU load as metric to decide scaling in or out operations. Beltran (2015) developed an automatic provisioning solution for multi-tier applications, called AutoMAP. The proposed mechanism is able to deal with both batch and interactive applications allowing horizontal and vertical scaling. The author created a definition, design and implementation of a new framework to automatically provision the virtual resources needed to execute a multi-tier application to meet the user performance requirements and to minimise the number of provisioned VMs. Jahn et al. (2015) present an optimal and centralised method to allocate resources to software-pipelined applications. To diminish the negative effects of unreliable processing units, their study shows how self-organisation can effectively restore the integrity of such a hierarchy when it is corrupted by a failing core.

In general we can say that the elasticity of cloud computing is an approach widely used for applications that require either high performance or reduction in the costs related to resource allocation. Table 1 presents a summarising grid regarding the initiatives discussed in this section. Here we can visualise two main gaps to explore:

1 considering initiatives that target pipeline applications, none of them explores monitoring and resource elasticity at independent manner for each individual stage of the application

2 all studied approaches suffer from the time penalties related to scaling out operations, where the application freezes up to completely delivering new resources.

In particular, in 2 , the presented initiatives often try to minimise resource allocation as much as possible to reduce the aforesaid impact in the application execution. 


\section{Pipel proposal}

This section presents Pipel - a model designed to provide automatic elasticity for pipeline-structured applications that run in the cloud. Subsection 3.1 presents design decisions of the proposed model. Subsection 3.2 shows the Pipel architecture, while Subsection 3.3 presents how we are addressing cloud elasticity. Particularly, these two last subsection highlight the Pipel's scientific contributions.

\subsection{Design decisions}

The development of a model that extracts the benefits of cloud elasticity is not a trivial task. The application should be designed to be partitioned into several different nodes and VMs, in addition to remain always running even when a resource is consolidated. Sometimes, coding an application to accomplish this requires user expertise about the application field itself, programming language and cloud provider application program interface (API). Thus, we designed Pipel to provide horizontal and reactive elasticity totally transparent and automatic at the application development viewpoint. The user submits its application to the cloud and compiles it with the Pipel middleware, so Pipel transforms a non-elastic application in a elastic one. Scaling in and out operations take place at runtime without user intervention and without any modification of the application beforehand. Pipel manages the addition and the removal of VMs, presenting an elasticity monitoring engine for each stage of the pipeline application. Below we summarise the design decisions that drove the Pipel's development:

- elasticity management at each stage of the pipeline application

- load balancing among the VMs belonging to a particular stage

- asynchronous communication strategy between Pipel components

- although the pipeline stages can present different IO and CPU requirements, all VMs that encapsulate them run over homogeneous resources, i.e., resources that present the same hardware configuration

- Pipel acts at the PaaS level of a cloud, allowing non-elastic pipeline applications to obtain the benefits of elasticity without the need for users' intervention.

\subsection{Architecture}

Figure 1 illustrates the Pipel architecture. Pipel is dimensioned to process only applications organised as a pipeline. The workflow includes a collection of stages, each one with its manager named stage controller (SC) and a set of worker VMs running worker processes. SC is responsible for receiving the processing requests and distributing them to be processed in the worker VMs. Each stage has a particular number of VMs in operation, allowing distribution of tasks among them. Tasks mean input requests that must be processed along the stages of the pipeline. All stages are monitored periodically by the elasticity manager (EM). According to the established rules and lower and upper thresholds in the EM, Pipel performs resource reorganisation actions to support the current demand over each stage of the application. Although EM is presented outside the cloud in Figure 1, it can be placed inside the cloud without problem. This is possible through the use of an API of the cloud provider, which requires only network connection to control the resources.

\subsubsection{Asynchronous multielasticity}

Concerning the fact that EM, not the application itself, increases or decreases the number of resources brings the following benefit: the application is not penalised by the overhead of synchronous allocation or deallocation of resources. Nevertheless, this asynchronism leads to a question: How does the system notify the application about the resources reconfiguration? To answer this question, Pipel provides a shared data area to enable communication between SCs and the EM. This area is private to the VMs inside the cloud. It can be implemented, for example, through NFS, message-oriented middleware (such as JMS or AMQP) or tuple spaces (such as JavaSpaces). The use of a shared area for interaction among VMs is a common practice when addressing private clouds (Wen et al., 2012; Cai et al., 2012).

The elasticity is guided by actions, numbered from 1 to 3 , that are enabled through the use of the shared area. To write and read elasticity actions to and from the shared area, EM uses a remote secure copy tool that targets a shared file system partition in the cloud front-end. When performing an scaling out operation, the manager monitors the procedure status up to the complete bootstrap of the VM template of a particular stage. Only after this confirmation, it writes action 1 to the shared area. after reading a notification for action 1, the $\mathrm{SC}$ of the corresponding stage can establish connections with the new worker VM. Both scaling out and scaling in operations (increase/decrease the number of VMs) are asynchronous from the application perspective because of SCs and Worker VMs can continue their execution normally.

In scaling in operations, instead of consolidating VMs automatically, this procedure is conducted through two actions. First, EM writes action 2 in the shared area asking for a permission to consolidate a particular set of worker VMs. Second, SC reads such action for its VMs, performs a series of disconnections to the mentioned VMs and writes action 3. This action is the final permission to consolidate these VMs. Particularly, action 2 is appropriate for the following reasons:

1 not finalising the execution of a process during its processing phase

2 to ensure that the application as a whole is not aborted with a sudden interruption of one or more processes. 
Figure 1 Pipel architecture (see online version for colours)

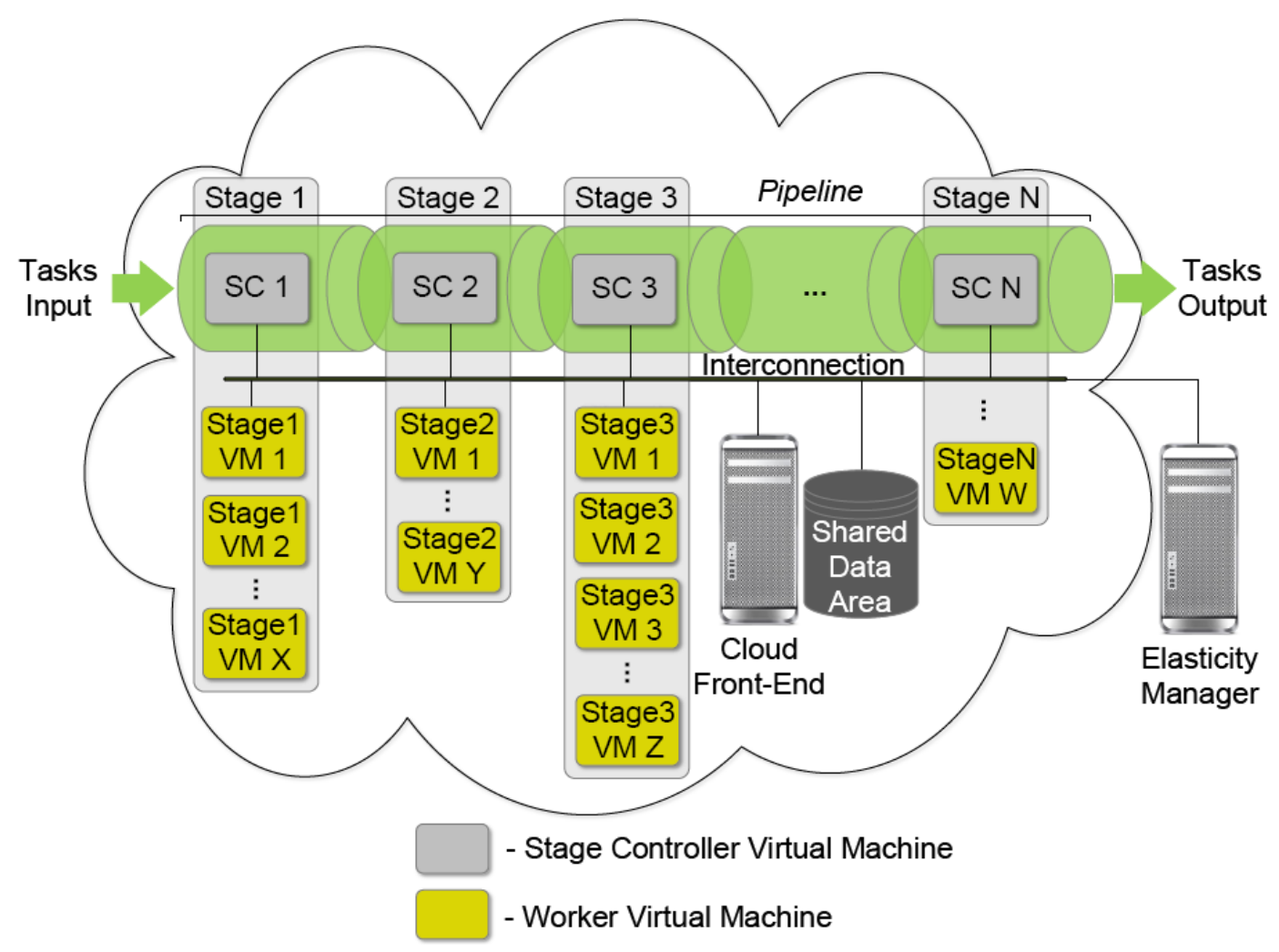

Figure 2 Example of the asynchronous multielasticity concept, emphasising the procedures with a single stage

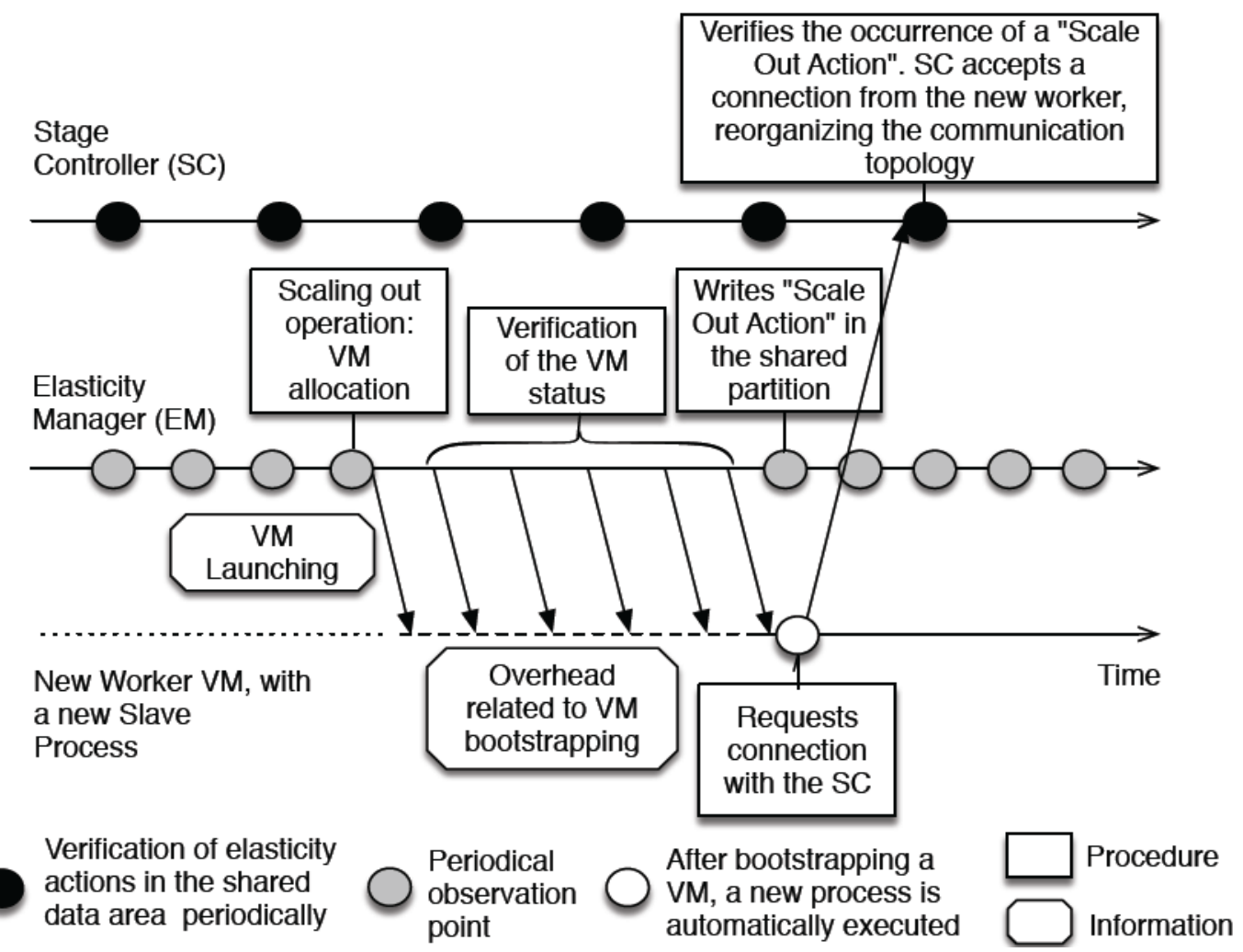


The second reason is important for a MPI application running over a TCP/IP network, since the application is interrupted as soon as detecting the premature death of any process.

Pipel introduces in the literature the concept of asynchronous multielasticity, which refers to the idea of non-blocking each stage of the application when different elasticity actions take place. This concept is viable due to the existence of a shared data area between the management module EM and the SCs. Figure 2 depicts an example of the asynchronous multielasticity concept, but involving a single stage for sake of simplicity. However, in a real situation we will have several SCs, one per stage, that interact with EM by the shared data area. Thus, multiple elasticity actions can take place in parallel, but never blocking the application up to completely delivering the resources.

\subsubsection{Detailing SCs and VM deployment}

A SC is in charge of managing the stages to which it is assigned. Its functions are:

1 receive the incoming tasks

2 store them in a queue

3 dividing each task for $\mathrm{n}$ worker VMs available on its stage

4 wait until all results return

5 aggregation of the results of all worker VMs

6 pass the results of the tasks to the next stage

7 visit the shared data area to control elasticity actions.

Figure 3 SC functions (see online version for colours)

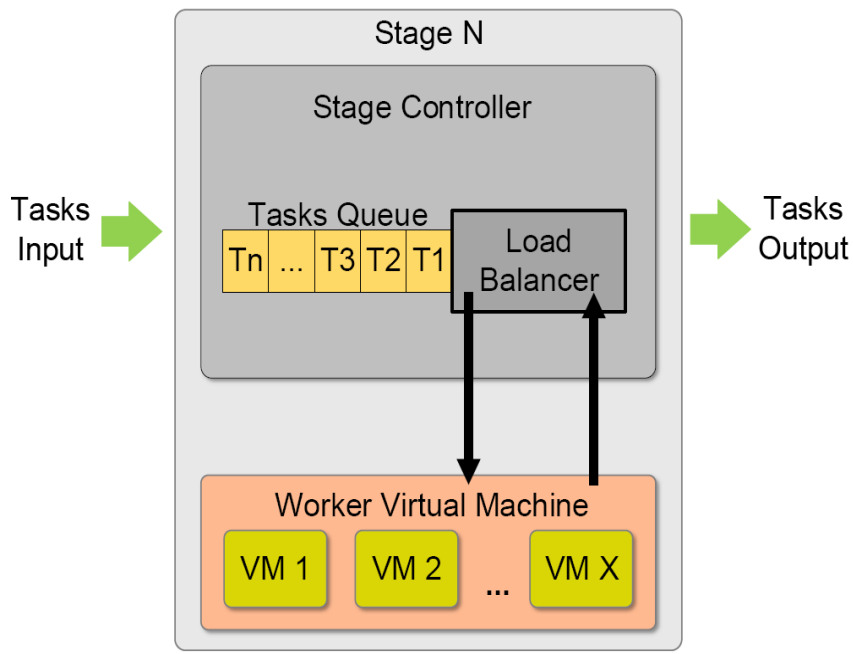

Figure 3 depicts the functioning of SC. Several works explore the parallel computing model master/slave in cloud computing (Righi et al., 2015; Kumar et al., 2014; Tran et al., 2015; Guoxin et al., 2015). In this scenario, the SC is the master and the worker processes running inside the worker VMs are slaves. We modelled a single template for worker VMs, where a parameter indicates the stage that an instantiation of this VM will operate. Each worker VM receives only one task at a time, representing the synchronous processing engine of a stage of the pipeline. At each VM instantiation, this new VM is assigned to a specific stage. Once it is allocated for stage 1, for instance, it will only process requests of the $\mathrm{SC} 1$ until it is consolidated or transferred to another stage. Finally, we have $\mathrm{n}$ templates for SC controllers, where $\mathrm{n}$ refers to the number of stages in the pipeline application.

\subsection{Elasticity management}

Pipel uses the concept of horizontal, automatic and reactive elasticity (Galante and de Bona, 2012; Herbst et al., 2013). We opted for horizontal elasticity because of vertical elasticity is limited to the resources available in a single computational node, in addition to the fact that the most of the operating systems do not allow resource resizing at runtime (Righi et al., 2015; Dutta ety al., 2012). Reactive comes from the use of load thresholds, one lower and another upper, which guide elasticity actions once exceeded. Automatic refers to both effortless and interventionless way of providing elasticity, which happens without adding any line of code in the pipeline application.

Pipel includes a management module (EM in Figure 1) to observe the behaviour of allocated worker VMs, providing elastic actions necessary to the current moment of the execution. Users can enter parameters in the SLA containing the maximum and minimum number of VMs. If the user does not enter an SLA, the application starts with 1 worker VM for each stage. Equation (1) sets the minimum number of VMs (minVM) required to run the application: here, $q$ denotes the number of application stages. Here, we multiply $q$ by 2 because of each stage has at leat two VMs, one to execute the tasks and other to run the SC. Equation 2 defines the maximum number of $\mathrm{VMs}$ on a particular moment to support the execution, where $q_{n}$ is the number of physical nodes and cores( $i)$ refers to the number of processing cores available on each physical node $i$.

$$
\begin{aligned}
& \min V M=q \times 2 \\
& \max V M=\sum_{i=0}^{q_{n}} \operatorname{cores}(i)
\end{aligned}
$$

Since the stages are monitored individually, each one can have different elasticity actions. While one is instantiating more VMs, another may consolidate them. In addition, we also check if there is a need to consolidate VMs in one stage, while another stage needs to instantiate a new VM. If this occurs the stage that intends to consolidate a VM pass it over to the stage that intends to instantiate a new one, saving both the bootstrap time related to the operating system and network transfer time of the VM template from the repository to the target node. This strategy saves time and money, since some cloud operators charge for the bootstrap of a new VM, in addition to charging for the full hour every time that a VM is instantiated.

Pipel decides about to apply or not resource elasticity for each stage based one two metrics: CPU load and 
conclusion time. EM periodically monitors them along the application execution. First, EM captures the CPU load of each VM, storing this data locally to be used latter in a time series calculus. Here we will observe if there is an under-or over-provisioning state, so adding or removing VMs to a particular stage to bypass such a situation. Second, EM captures the conclusion time of each stage, so comparing this data about each stage to drive resource reorganisation. A key feature to maintain performance in a pipeline application refers to the cadence among the stages, so here we will add or subtract VMs to a particular stage to provide a homogeneous processing rhythms among the stages as close as possible. In this, way, we organised the next subsections to explain how we are addressing CPU load and execution time, so presenting in Subsection 3.2.3 our combination of such information to drive elasticity actions.

\subsubsection{CPU load of VMs at each stage}

Pipel computes the CPU load of worker VMs of a particular stage e through a function named general processing cargo (GPC) [see equation (4)]. This equation receives two parameters, $o$ and $e$, returning a simple arithmetic average of the workloads of $q_{e} \mathrm{VMs}$ in the $o^{\text {th }}$ monitoring observation of stage $e$. Equation (4) calls equation (3) using three parameters, where $S E S$ refers to the CPU load of the VM $i$ belonging to stage $e$ at the $o^{\text {th }}$ observation. Equation (3) computes the CPU load as a simple exponential smoothing (SES) considering the last captured CPU load value and the others up to the first one. Thus, we give more weight to the newest observation but we also consider historical observations. After obtaining the value of $\operatorname{GPC}(o, e)$, we use it to compare with the upper and lower thresholds to trigger or not elasticity actions to support the current demand over the stage number $e$.

$$
\begin{aligned}
& \operatorname{SES}(i, o, e)= \begin{cases}\frac{C P U(i, o, e)}{2} & \text { if } o=0 \\
\frac{\operatorname{SES}(i, o-1, e)}{2}+\frac{C P U(i, o, e)}{2} & \text { if } o \neq 0\end{cases} \\
& \operatorname{GPC}(o, e)=\frac{\sum_{i=0}^{q_{e}} \operatorname{SES}(i, o, e)}{q_{e}}
\end{aligned}
$$

The use of this smoothing technique is pertinent to avoid VM trashing (Bryant et al., 2011), i.e., false-positive or false-negative operations on VM scaling. Figure 4 illustrates situations of peak loads, where a single CPU load would launch an erroneous resource reorganisation, but our $S E S$ metric prevents it. A false-positive situation happens when an elasticity action is triggered after detecting a sudden load peak that exceeds one of the thresholds. We can see falsenegative situations mainly on elasticity points that trigger resource reorganisation when finding one of two conditions: after exceeding one of the thresholds either for a predefined number of consecutive observations or for a predefined time. On both situations, elasticity can be pertinent to adjust the load but it does not happen, or it is delayed. Our SES solution surpasses the aforementioned ideas, taking into account that it considers aging and the historical behaviour of the application.

Figure 4 CPU load with and without time series technique (see online version for colours)

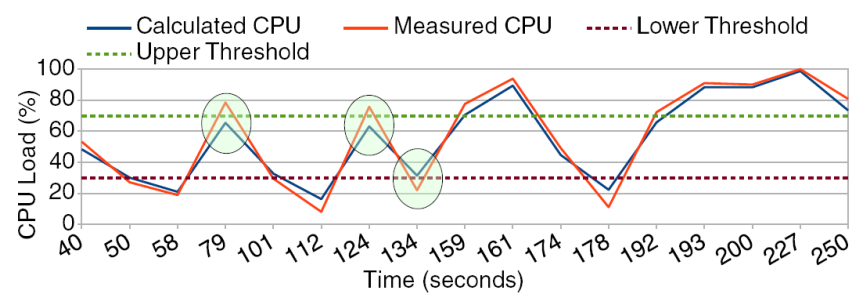

\subsubsection{Conclusion time of each stage}

EM also monitors execution times for each stage to maintain a close cadence among them. This is crucial for performance in pipeline applications, since a slower stage can compromise the entire execution. Considering that the stages can present heterogeneous processing requirements, the idea here is to provide more resources for time-consuming stages. Communication between stages is done asynchronously; so EM needs to observe each stage separately, but it also has a method that analyses the continuity of the application in general. In this way, Pipel incorporates two conditions as can be seen in equations (5) and (6):

1 the execution time of an stage $(t(e))$ is 1.5 times greater than the arithmetic average of the execution times of all $n$ stages

2 the execution time of a stage is shorter than the half of the arithmetic average of the execution times of all stages.

$$
\begin{aligned}
& t(e)>\frac{3}{2} \times \frac{\sum_{i=1}^{n} t(i)}{n} \\
& t(e)<\frac{1}{2} \times \frac{\sum_{i=1}^{n} t(i)}{n}
\end{aligned}
$$

\subsubsection{Cloud elasticity decision making}

Our elasticity strategy combines CPU load and conclusion time by defining four conditions and two actions, as presented in Table 2. The conditions are evaluated by EM periodically, each time the elasticity testing take place. These conditions and actions are used to drive elasticity decision making as we can se in Algorithm 1. Here, we can emphasise two aspects:

1 we are addressing elasticity at each stage individually

2 in line 7 we have a scaling out operation, but the application continues its normal execution up to completely delivering the new resource, as discussed in the asynchronous multielasticity concept (see Subsection 3.2.1). 
Table 2 Conditions and actions used in Pipel monitoring routine

\begin{tabular}{ll}
\hline Condition/action & \multicolumn{1}{c}{ Description } \\
\hline Condition1(e) & $\begin{array}{l}\text { Arithmetic average of CPU load [see } \\
\text { equation (4)] from all VMs belonging to } \\
\text { stage e is greater than the upper threshold }\end{array}$ \\
Condition2(e) & $\begin{array}{l}\text { Arithmetic average of CPU [see equation (4)] } \\
\text { from all VMs belonging to stage e is lower } \\
\text { than the lower threshold }\end{array}$ \\
& $\begin{array}{l}\text { Conclusion time of the last execution of stage } \\
\text { e is greater than the arithmetic average time } \\
\text { of all stages multiplied by 1.5 [see equation } \\
\text { (5)] }\end{array}$ \\
Condition3(e) & $\begin{array}{l}\text { e is shorter than the half of the arithmetic } \\
\text { average of the conclusion time of all stages }\end{array}$ \\
& $\begin{array}{l}\text { Add a VM on stage e; } \\
\text { Remove a VM from stage e; }\end{array}$ \\
Action1(e) & Action2(e)
\end{tabular}

\section{Evaluation methodology}

To evaluate Pipel, first we developed a prototype that runs over OpenNebula private cloud (version 4.14). We developed Pipel using Java due to its compatibility with the XML-RPC protocol, which was incorporated by OpenNebula. This API is in charge of enabling both monitoring and elasticity actions, where EM acts as a client and cloud front-end acts as a server. To accomplish communication among stages, Pipel uses a shared data through network file system (NFS). This data area is available to both EM and SCs. It also aims to store application execution history such as execution times of the stages, which VMs are allocated to each stage, application data and monitoring logs. We start the application with a single worker VM per stage, with monitoring periods of $5 \mathrm{~s}$. In addition, we are using $30 \%$ and $70 \%$ for the lower and upper thresholds, respectively. We selected these values because of they are commonly used in distributed cloud-based parallel applications (Righi et al., 2015; Al-Haidari et al., 2013). In particular, they will be used to handle elasticity based on CPU load, as depicted in Subsection 3.3.1 and 3.3.3.

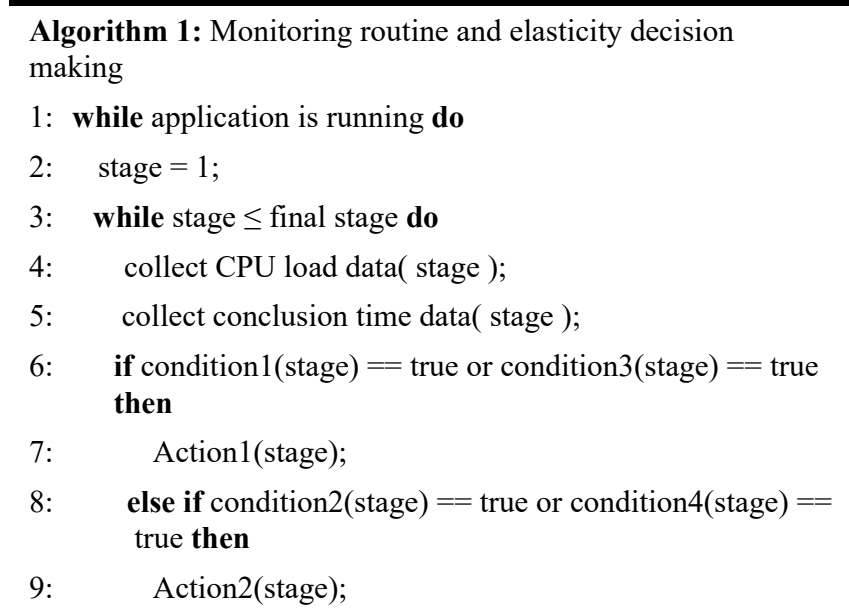

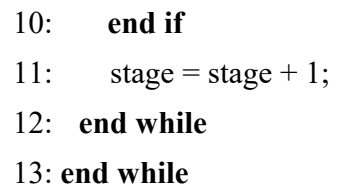

Regarding the cloud infrastructure, we work with 8 quad-core nodes, all of them with i5 processors. All computers have the same configuration, and therefore considered as a homogeneous computing environment. The first node runs the cloud front-end, while the second runs EM. The six nodes remaining are available to run the application; so it has the ability to run up to 24 VMs. Each stage needs at least one VM to run a SC and another to run a worker process. All nodes are interconnected with a fast Ethernet local network, considering both network interface cards and switch.

\subsection{Application}

We developed a pipeline application that addresses images as input tasks and computational graphic transformations as processing core at each stage. We based our experimentation on the Jie's ( $\mathrm{Li}$ et al., 2010) work: large-volume satellite data is loaded from specific repositories at different resolutions, using algorithms to find scientific importance of variables and generate tables with all information and deliver the results to scientist analysis. In this study a similar application was created with three image processing stages:

1 Greyscale: a colour in the red, green and blue (RGB) model can be described by indicating the amount of RGB that contains. In this step the image goes through an analysis pixel by pixel and the R, G and B have their values changed.

2 Colour reversal: a linear inverse mapping function, the contrast changes so that a dark area (low gray level values) is clear. The negative of an image in strips $R, G$ and $B$ image changes introduces intense hues, with the replacement of colours for their complementary.

3 Thresholding: the image at this stage has each point (RBG) analysed. It is given a threshold and each point is checked, if it is below the threshold is changed to $(0,0,0)$ (black) and if above is changed to $(255,255,255)$ (white).

After crossing by the three stages, an image is completely transformed and this task can be classified as done. Considering that the processing applied to the images is data-oriented parallelism, each incoming image arriving in a particular stage is decomposed in accordance with the number of worker VMs belonging to this stage. After that, SC distributes these sub-parts to be processed in parallel, collecting and aggregating the results afterwards. Finally, the task is done, being able to be passed to the next stage.

The input stream for pipeline applications can be intense, erratic or irregular. Thus, to test the system in different workloads we modelled four different intensities: 
increasing, decreasing, constant and oscillating. The difference among them is the sequence of images; more precisely, their size. The larger the figure size, the larger the time to conclude a stage. The sum of all the images in each workload configuration results in the same number (420,000,000 pixels). So, in all tests the amount of data processed by the application is the same. Finally, the input images were collected from two web sites: HUBBLE2 and NASA3. They provide open images from telescopes and satellites in $4 \mathrm{k}$ high resolution.

\subsection{Evaluation scenarios}

Aiming at evaluating Pipel, we modelled two scenarios: (s1) without elasticity and; (s2) with elasticity. In the first, the application runs with all the different processing loads without the use of the features of the EM. Thus, allocations and consolidation of resources are not enabled. In this scenario we use three SCs (one for each stage) and three worker VMs (one for each stage). In the second scenario, we are enabling the Pipel's elasticity algorithm. As in the first scenario, the second also starts with $6 \mathrm{VMs}$ but it can enlarge this number up to 10 .

\subsection{Metrics}

We are using four metrics to evaluate Pipel over the two scenarios and four workloads. First, we are defining two novel metrics named elastic speedup (ES) and elastic efficiency (EE). Second, we are using the Amazon concept of billing to determine the energy consumed by the application. Third, we present a notion of cost based on the principles of parallel computing. In particular, this last is pertinent to decide about elasticity viability.

\subsubsection{ES, EE and resource consumption}

Both ES and EE are explored here in accordance with horizontal elasticity, in which the instances can scale either out or in with the application demands. Furthermore, we consider a homogeneous system in which each VM runs in $100 \%$ of a CPU core, regardless of the cloud level (IaaS, PaaS or SaaS). ES is calculated by the function $\operatorname{ES}(n, l, u)$ according to equation (7), where $n$ denotes the initial number of VMs and $l$ and $u$ represent the lower and upper bounds for the quantity of VMs, respectively. $t_{n e}$ and $t_{e}$ refer to the conclusion times of an application that was executed without and with elasticity support, respectively. Here, $t_{n e}$ is obtained with the lowest possible number of VMs, in our case $l$, providing an analogy with sequential execution in the standard speedup.

$$
E S(n, l, u)=\frac{t_{n e}(l)}{t_{e}(n, l, u)}
$$

where $l \leq n \leq u$.

Function $E E(n, l, u)$ in equation (8) computes EE. Its parameters are the same as the preceding function ES. Efficiency represents how effective the use of resources is, being the entity positioned last as denominator in the formula. In addition, the resources here are malleable; therefore, we designed a mechanism to reach a single value for them coherently. To accomplish this, EE assumes the execution of a monitoring system that captures the time spent on each configuration of VMs. Equation (9) indicates use of the resources, where $p t_{e}(i)$ is the application's partial time when running over $i$ VMs. Logically, if elasticity actions do not occur, $p t_{e}(i)$ and $t e(n, l, u)$ values will be identical for $i=n$. Equation (8) presents parameter $\mathrm{n}$ in the numerator, which is multiplying the ES. More precisely, this occurs because $\operatorname{ES}(n, n, n)$ is always equal to 1 and $\operatorname{Resources}(n, n, \mathrm{n})$ equal to $n$, so $n$ in the numerator returns an EE of $100 \%$.

$$
E E(n, l, u)=\frac{E S(n, l, u) \times n}{\operatorname{Resource}(n, l, u)}
$$

where

$$
\operatorname{Resource}(n, l, u)=\sum_{i=l}^{u}\left(i \times \frac{p t_{e}(i)}{t_{e}(n, l, u)}\right)
$$

The denominator in equation (8) was added to capture each infrastructure configuration (from $l$ to $u \mathrm{VMs}$ ) and its participation in the entire execution, presenting the sum of the partial values as the final value for $\operatorname{Resource}(n, l, u)$. The use of a flexible number of resources helps to provide better parallel efficiency when comparing elastic and non-elastic parallel executions. At a glance, there are two approaches to make viable the aforementioned statement:

1 the elastic execution presents an equal, or even a bit greater, execution time when compared with a non-elastic configuration, but we employ a smaller set of resources to reach the first case

2 even devoting a larger number of resources because the CPU-bound HPC application demands them, the gain in the time perspective outperforms the investment in resources.

Although scaling in is a key operation to accomplish 1, scaling out is essential for 2 .

\subsubsection{Energy}

The energy metric relies on the close relationship between energy and resource consumption as presented by Orgerie et al. (2014). We are using equation (10) to create an energy index, with $l$ and $u$ with the same meanings described in Subsection 4.3.1. Here, we use the same ideas of the pricing model employed by Amazon and Microsoft; they consider the number of VMs at each unit of time, which is normally set to an hour. $p t_{e}(i)$ presents the time that the application executed with $i$ VMs. Therefore, our unit of time depends on the measure of $p t_{e}$ (in minutes, seconds or milliseconds, and so on) in which the final intent is to sum the number of VMs used at each unit of time. 


$$
\operatorname{Energy}(l, u)=\sum_{i=l}^{u}\left(i \times p t_{e}(i)\right)
$$

For example, considering a unit of time in minutes and an application completion time of $7 \mathrm{~min}$, we could have the following histogram: $1 \mathrm{~min}(2 \mathrm{VMs}), 1 \mathrm{~min}(2 \mathrm{VMs}), 1 \mathrm{~min}$ (4 VMs), $1 \mathrm{~min}(4 \mathrm{VMs}), 1 \mathrm{~min}(2 \mathrm{VMs}), 1 \mathrm{~min}(2 \mathrm{VMs})$ and $1 \mathrm{~min}(2 \mathrm{VMs})$. Finally, $2 \mathrm{VMs}$ were used in $5 \mathrm{~min}$ (partial resource equal to 10 ) and $4 \mathrm{VMs}$ in $2 \mathrm{~min}$ (partial resource equal to 8), summing to 18 for equation (10). Thus, equation (10) analyses the use from $l$ to $u \mathrm{VMs}$, considering the partial execution time on each infrastructure size. To the best of our understanding, the energy index here is relevant for comparisons among different elastic-enabled executions by varying $l$ or $u$.

\subsubsection{Cost}

We use a cost model to estimate elasticity viability by multiplying the application time by the energy metric. Our notion of cost in equation (11) represents an adaptation of the cost of a parallel computation (Wilkinson and Allen, 2005), now to support elastic environments. We only change the number of processors by the value obtained from one of the energy models. The final idea consists of obtaining a better cost when enabling the Pipel's elasticity feature in a comparison with an execution of a fixed number of processes. In other words, a configuration solution may be classified as bad if it is capable of reducing the execution time by one-half with elasticity but spends four times more energy, thus increasing costs. Therefore, considering the values of the cost function in elastic and non-elastic environments, the objective is to preserve the truth of Inequality 12 .

$$
\begin{aligned}
& \text { Cost }=\text { App_Time } \times \text { Energy then, the goal is to obtain } \\
& \operatorname{Cost}_{\alpha} \leq \operatorname{Cost}_{\beta}
\end{aligned}
$$

where $\alpha$ and $\beta$ refer to Pipel execution with and without elasticity support, respectively.

\section{Results}

In this section, we present the evaluation results of Pipel when considering the aforementioned scenarios, workloads and metrics. Firstly, we present an analysis of time, energy and cost in Subsection 5.1. Results about ES and elasticity efficiency are detailed in Subsection 5.2. Focusing on analysing the cloud behaviour, we selected some moments to discuss and illustrate the history of resource allocation in Subsection 5.3, so presenting why elasticity actions took place. In Subsection 5.4, we show resource allocation profiles that are useful to visualise the use of different VM deployments along the time.

\subsection{Observing time, energy and cost}

To analyse the metrics time, energy and cost, Table 3 presents all results obtained from executions of the four workloads in two different scenarios: (s1) running with the minimum amount of resources without the elasticity feature; (s2) starting the execution with the minimum amount of resources with the elasticity feature enable. We also computed these metrics in each pipeline stage to present them separately in the table.

Analysing time, all executions from scenario s2 achieved better results than the ones from scenario s1 where elasticity was disabled. It shows that the more resources available to distribute load the faster is the application execution, increasing performance in each pipeline stage. Observing separately this metric in each stage, the stage 1 achieved best time in all workloads and scenarios when comparing with the stages 2 and 3. On the other hand, in the same comparison the stage 3 obtained the worst time showing that, although the workload was different along time, the load was the same for all executions. Looking at the results from both scenarios, we can observe that the overall execution time of the application reduced 38\% (increasing), 24\% (decreasing) 33\% (constant), and 35\% (oscillating) when enabling elasticity for resource reorganisation. In other words, the increase of resources in a asynchronous way allowed the application to accelerate its execution by using new resources only when they were available. With a better load distribution between more worker VMs, it was possible to the application achieve better performance.

At the energy metric perspective, the gains of performance from scenario s2 resulted in more resource usage. As a result, in this scenario the energy increased 15\% (increasing), 18\% (decreasing), 6\% (constant) and 4\% (oscillating) when compared to scenario s1. However, the gains of performance were greater than the waste of resources showing that a large waste of resources can result in a even larger gain in performance. The metric cost considers both metrics to evaluate different executions using a single value. In this way, the cost of all executions in scenario s2 achieved lower values than the ones of the same workloads in scenario s1. It resulted in decreases of $24 \%$ (increasing), 33\% (decreasing), 35\% (constant) and 33\% (oscillating) in the cost metric in favour of the use of elasticity. These results show that a higher use of resources do not impact negatively in the final cost of the application. Particularly, the gains of execution time are enough to surpasses the waste of resources in the total cost. 
Table 3 Analysis of time, energy and cost metrics in the four workloads

\begin{tabular}{|c|c|c|c|c|c|c|c|c|c|c|c|c|c|}
\hline \multirow{3}{*}{ Scenario } & \multirow{3}{*}{ Stage } & \multicolumn{12}{|c|}{ Application workload } \\
\hline & & \multicolumn{3}{|c|}{ Increasing } & \multicolumn{3}{|c|}{ Decreasing } & \multicolumn{3}{|c|}{ Constant } & \multicolumn{3}{|c|}{ Oscillating } \\
\hline & & Time & Energy & Cost & Time & Energy & Cost & Time & Energy & Cost & Time & Energy & Cost \\
\hline \multirow[t]{4}{*}{ s1 } & 1 & 1,767 & 3,534 & 6,244 & 1,715 & 3,430 & 5,882 & 1,729 & 3,458 & 5,978 & 1,786 & 3,572 & 6,379 \\
\hline & 2 & 1,817 & 3,634 & 6,602 & 1,722 & 3,444 & 5,930 & 1,739 & 3,478 & 6,048 & 1,805 & 3,610 & 6,516 \\
\hline & 3 & 1,870 & 3,740 & 6,993 & 1,743 & 3,486 & 6,076 & 1,777 & 3,554 & 6,315 & 1,829 & 3,658 & 6,690 \\
\hline & Total & 1,870 & 10,908 & 20,397 & 1,747 & 10,360 & 18,057 & 1,777 & 10,490 & 18,640 & 1,829 & 10,840 & 19,826 \\
\hline \multirow[t]{4}{*}{ s2 } & 1 & 1,080 & 4,120 & 4,449 & 1,198 & 3,460 & 5,208 & 1,142 & 3,950 & 4,510 & 1,109 & 4,109 & 4,556 \\
\hline & 2 & 1,129 & 4,201 & 4,742 & 1,222 & 4,018 & 4,909 & 1,177 & 3,644 & 4,288 & 1,133 & 3,575 & 4,050 \\
\hline & 3 & 1,154 & 4,259 & 4,914 & 1,328 & 4,348 & 5,774 & 1,184 & 3,572 & 4,229 & 1,172 & 3,603 & 4,222 \\
\hline & Total & 1,154 & 12,580 & 14,517 & 1,328 & 12,319 & 16,359 & 1,184 & 11,166 & 13,220 & 1,172 & 11,287 & 13,228 \\
\hline
\end{tabular}

Note: For better presentation, we do not show the last three digits of the cost values.

Table 4 Analysis of resource metrics (RE), ES and EE in the four workloads loads

\begin{tabular}{|c|c|c|c|c|c|c|c|c|c|c|c|c|c|}
\hline \multirow{3}{*}{ Scenario } & \multirow{3}{*}{ Stage } & \multicolumn{12}{|c|}{ Application workload } \\
\hline & & \multicolumn{3}{|c|}{ Increasing } & \multicolumn{3}{|c|}{ Decreasing } & \multicolumn{3}{|c|}{ Constant } & \multicolumn{3}{|c|}{ Oscillating } \\
\hline & & $R E$ & $E S$ & $E E$ & $R E$ & $E S$ & $E E$ & $R E$ & $E S$ & $E E$ & $R E$ & $E S$ & $E E$ \\
\hline \multirow[t]{4}{*}{ s1 } & 1 & 1 & 1 & 1 & 1 & 1 & 1 & 1 & 1 & 1 & 1 & 1 & 1 \\
\hline & 2 & 1 & 1 & 1 & 1 & 1 & 1 & 1 & 1 & 1 & 1 & 1 & 1 \\
\hline & 3 & 1 & 1 & 1 & 1 & 1 & 1 & 1 & 1 & 1 & 1 & 1 & 1 \\
\hline & Total & 3 & 1 & 1 & 3 & 1 & 1 & 3 & 1 & 1 & 3 & 1 & 1 \\
\hline \multirow[t]{4}{*}{ s2 } & 1 & 3.81 & 2.68 & 0.70 & 3.63 & 2.05 & 0.56 & 3.46 & 2.29 & 0.66 & 3.71 & 2.59 & 0.70 \\
\hline & 2 & 3.72 & 2.59 & 0.70 & 3.29 & 1.99 & 0.60 & 3.10 & 2.18 & 0.71 & 3.16 & 2.54 & 0.80 \\
\hline & 3 & 3.69 & 2.63 & 0.71 & 3.27 & 1.72 & 0.53 & 3.02 & 2.25 & 0.75 & 3.07 & 2.44 & 0.79 \\
\hline & Total & 10.9 & 2.63 & 0.72 & 9.28 & 1.73 & 0.56 & 9.43 & 2.25 & 0.72 & 9.63 & 2.44 & 0.76 \\
\hline
\end{tabular}

\subsection{Analysing ES and EE metrics}

Table 4 shows the ES and EE results of all executions in scenarios s1 and s2. Additionally, the table also shows the metric RE used to compute EE. Due to reorganisation of resources involve only worker VMs in scenario s2, when computing EE in scenario s1 it is considered a single VM per stage. For this reason, the minimum amount of resources available for the application in scenario s1 is $3 \mathrm{VMs}$, been this value used when computing EE for a global analysis considering all stages. Therefore, all ES and EE results from table in scenario $s 1$ resulted in the value 1 because executions of this scenario are considered as base to compute ES and EE. In addition, ES and EE were designed to evaluate elastic executions that occurred only in scenario s2.

Focusing on scenario s2, the workloads increasing and Decreasing achieved the best and worst ES value, respectively. The main reason for these results is that in the Decreasing workload, the higher computation load occurs in the first half of execution when there are less resources available. On the other hand, in the increasing workload, when the computation load achieve the higher values there are more resources already available to handle it. These settings also obtained, respectively, best and worst values when evaluating time in Table 3 . This relation between metrics time and ES is due to ES considers in its computation values of the time metric. The same relation is true between metrics RE and time where the executions that obtained best/worst RE were the same that obtained best/worst performance. The metric EE, in turn, demonstrates how was the level of utilisation of the available resources in the entire execution. Particularly, the metrics ES and EE are focusing in compute the magnitude of performance gains and utilisation degree of the available resources. In general, the results in Table 4 show that a better performance is equivalent to a lower efficiency. Conversely, a better efficiency results in reduced gains in performance. The reason for this behaviour is that as the application used in experiments is characterised as CPU-intensive, the distribution of load between a larger set of VMs decreases the CPU level of all VMs. It results in a fast computation and resources not over-loaded.

\subsection{History of resource allocation}

Figures 5 to 12 detail all executions in both scenarios $\mathrm{s} 1$ and s2. Figures representing the system load and the used thresholds were generated using the same scale for all axis to facilitate comparison between different figures. In these subsection we analyse the workloads separately. 


\subsubsection{Increasing workload analysis}

Figure 5 presents traces of resource utilisation and allocation when running the application with the increasing workload. In the part (a) of the figure, the application was executed in scenario s1 without elasticity. The thresholds in this figure illustrate that even though the load violated one of the thresholds, no elasticity actions took place. The load variations during the time represent communication phases where data output from one stage is sent to the next one. During this period worker VMs wait until all new data is available for processing. On the other hand, in the part (b) of the figure, the increasing workload was executed with the elasticity enabled. In this case, the execution ended faster then scenario s1 since more resources were made available to the application. As more resources are added, the load level tends to stay inside the upper and lower thresholds.

Figure 5 History of resource allocation and resource utilisation level when running the application with the increasing workload in the two scenarios, (a) elasticity disabled (b) elasticity enabled (see online version for colours)

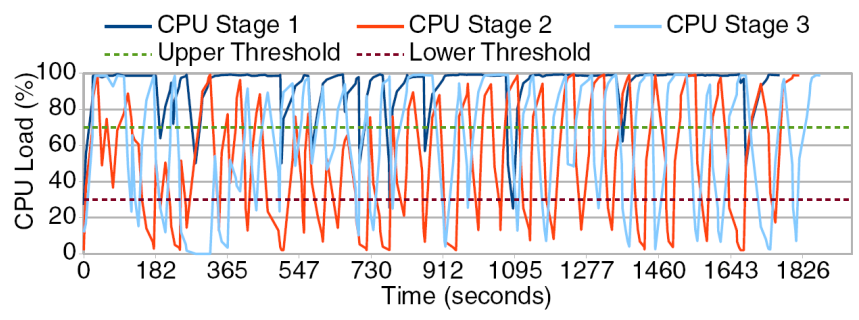

(a)

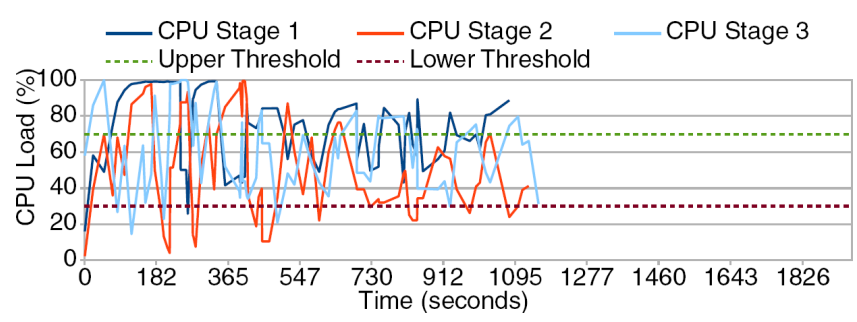

(b)

Figure 6 Resource allocation per stage running the increasing workload (see online version for colours)
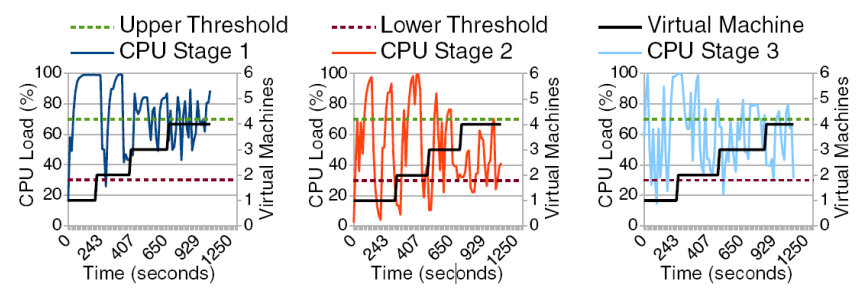

Concerning resource usage in each stage, Figure 6 shows separately the resource allocations that occurred when running the application with the increasing workload. In all stages, the amount of elasticity operations were the same where three extra VMs were added to each stage. This behaviour occurred because the load increases equally for all stages during the execution time. On the other hand, as the load increases constantly until the end of the execution, elasticity operations to remove resources did not take place. In addition, in stages 1 and 3, the load violated the upper threshold close to the execution end but any new VMs were added. It happened because the allocation processes started but the new VMs were available only after the application's ending.

\subsubsection{Decreasing workload analysis}

Figure 7 presents traces of resource utilisation and allocation when running the application with the Decreasing workload. In this workload, at the beginning of the execution the input data starts with larger images. Consequently, the application starts with high levels of processing in all stages. As a result, EM starts allocating new resources to handle this initial demand. Figure 7(b), shows the execution of this workload running the EM for resource reorganisation. A comparison between part (a) and (b) of the figure shows that running the application with elasticity allows the application to end its execution quicker than running the same application without elasticity.

Figure 7 History of resource allocation and resource utilisation level when running the application with the decreasing workload in the two scenarios, (a) elasticity disabled (b) elasticity enabled (see online version for colours)

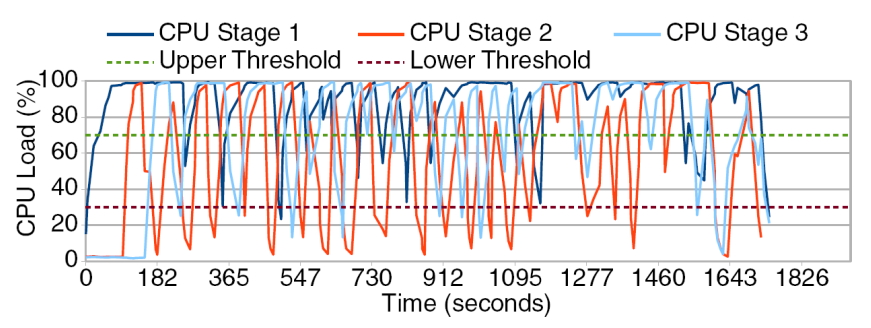

(a)

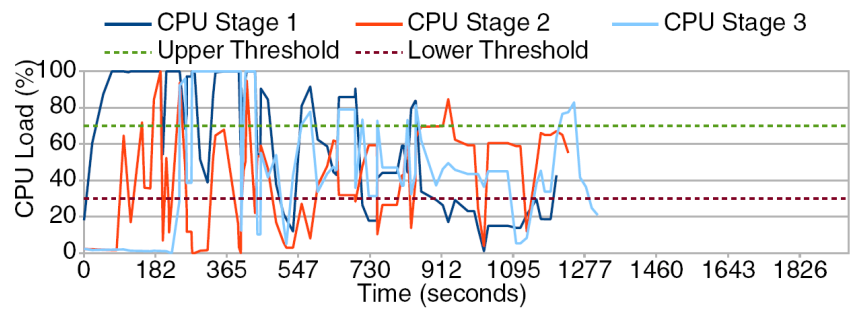

(b)

Figure 8 Resource allocation per stage running the decreasing workload (see online version for colours)

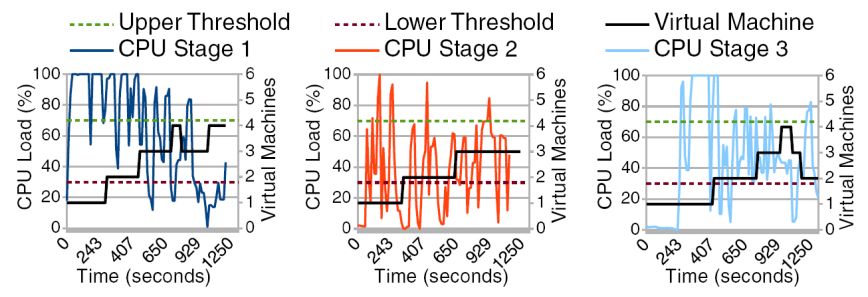

To analyse resource allocation in each stage, Figure 8 shows them separately. As the first stage is the only one that receives the initial load, the load level in stages 2 and 3 took some more time to grow. Consequently, resource 
reorganisations occurred in different ways in each stage. Differently from the increasing workload, in the Decreasing workload consolidation of resources took place. In particular, stage 1 registered a VM consolidation at the instant 826 seconds. However, at the instant 1,076 seconds the EM added again an extra VM in stage 1 to supply an upper threshold violation. Although it happened close to the end of the application execution, this allocation ended in time to deliver it to the application. Meanwhile, stage 3 registered $2 \mathrm{VM}$ consolidations in instants 1,087 and 1,152 seconds. In this case, these operations occurred close to the end of the application not allowing new resource allocations since there were no time enough to deliver it.

\subsubsection{Constant workload analysis}

Figure 9 presents traces of resource utilisation and allocation when running the application with the constant workload. This workload configuration is the most uniform of all. This means that, enabling elasticity, the processing levels tend to stay between the thresholds. Comparing executions without elasticity [Figure 9(a)] and with elasticity [Figure 9(b)], when running the application with elasticity the execution time was reduced by $33 \%$. Although the input load has the same size for all images, the figure shows fluctuations in the load as result from communications between stages. This behaviour is more visible when there are less resources available to the application where the size of the grain that each VM sends by network is greater.

Figure 9 History of resource allocation and resource utilisation level when running the application with the constant workload in the two scenarios, (a) elasticity disabled (b) elasticity enabled (see online version for colours)

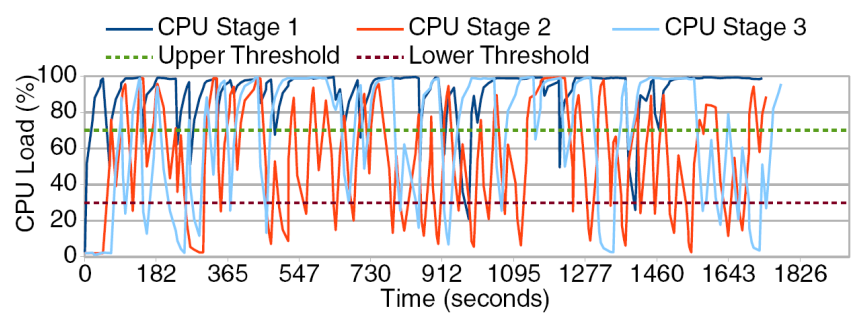

(a)

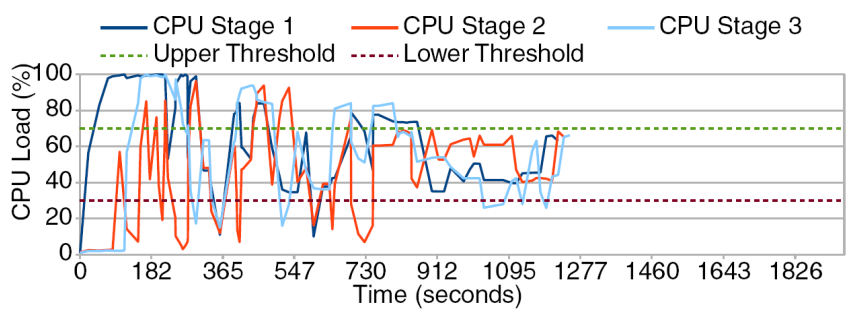

(b)
Figure 10 Resource allocation per stage running the constant workload (see online version for colours)

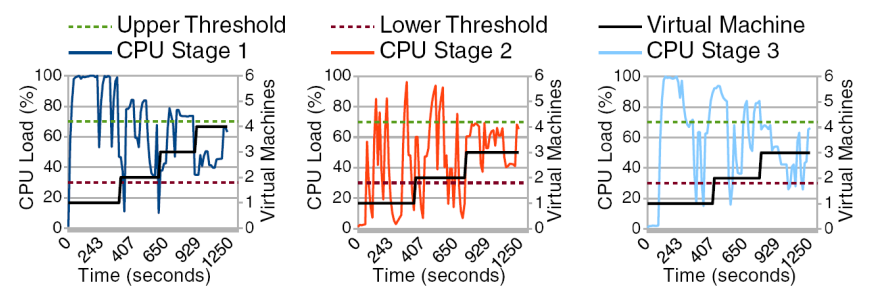

Considering stages individually, Figure 10 shows resource allocations in the three stages. The time each VM was added to each stage was similar. Besides, in stage 1 each new VM was available seconds before when comparing with stage 2 . The same behaviour is true comparing stages 2 and 3 where each new VM was available in stage 2 a few seconds before in stage 3. In particular, in stage 1 the EM added an extra $\mathrm{VM}$ in comparison with the other stages. Regarding resource deallocation, this workload behaviour remained the same all the time no requiring consolidation of resources.

\subsubsection{Oscillating workload analysis}

Figure 11 presents traces of resource utilisation and allocation when running the application with the oscillating workload. Confronting both parts (a) and (b) of Figure 11, the execution where resource reorganisations occurred achieved performance $35 \%$ better than when executing the same workload with a fixed amount of resources. The increase of resources in the elastic execution was responsible for this performance gain. More specifically, the increment of worker VMs in each stage favoured the load balancing and accelerated the computation of each phase. To illustrate these resource reorganisations, Figure 12 shows elasticity operations in each stage individually. In both stages 1, 2 and 3, EM managed the cloud resources increasing three VMs. As seen in the other workloads, new VMs were available in stage 1 before stages 2 and 3 . Particularly, it happens because the load always gets higher firstly in the first stages. For this reason, the stage 3 was the last one to receive input data and produce output data. Regarding decreasing of resources, just one scaling in operation occurred in this stage 3 . This operation took place close to the end of the execution when the load in stage 3 decreased below the lower threshold. At the same time, the load in stage 2 violated the upper threshold triggering a new VM allocation. However, the application ended its execution seconds later not been able to connect with this new resource. 
Figure 11 History of resource allocation and resource utilisation level when running the application with the oscillating workload in the two scenarios, (a) elasticity feature disabled (b) elasticity feature enabled (see online version for colours)

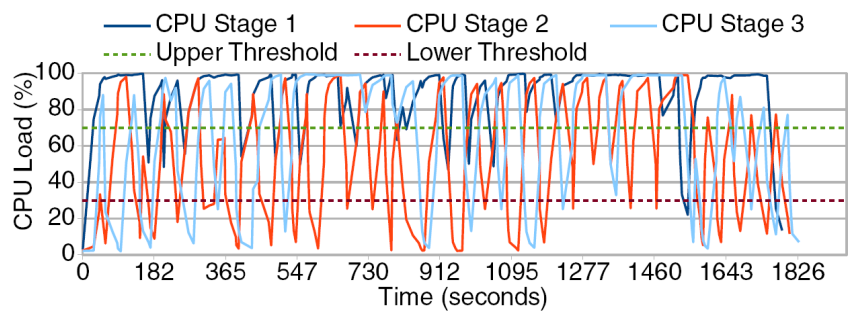

(a)

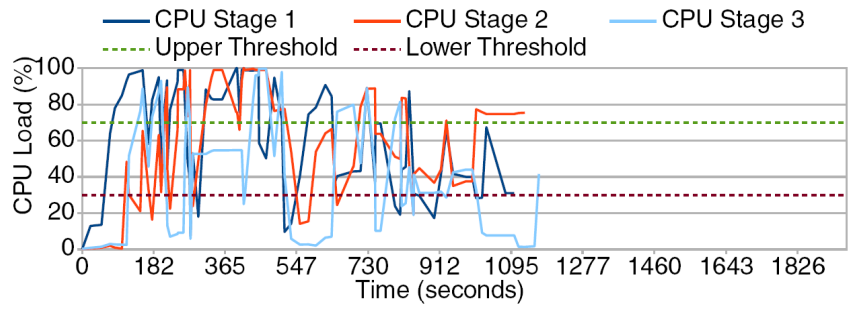

(b)
Figure 12 Resource allocation per stage running the oscillating workload (see online version for colours)

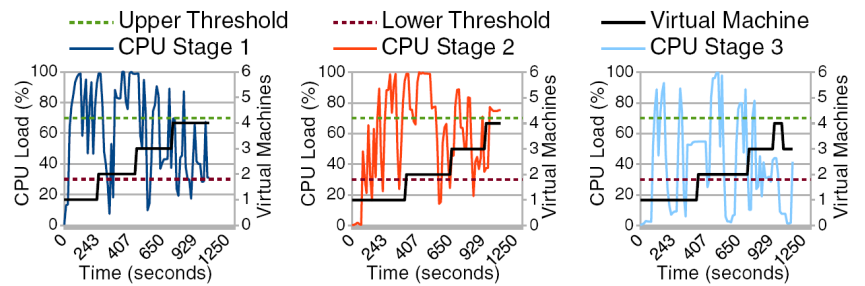

\subsection{Profiling the execution to observe resource consumption}

Figure 13 shows the assessment of estimated energy metric obtained in all scenarios and workloads. This metric represents the total amount of resources that were used throughout the application execution. In this subsection, the aim is to make resource allocation comparison considering only worker VMs. The SC VMs are not monitored during the application execution and for this reason we are not analysing them.

Figure 13 Allocation and consumption of resources on both scenarios (see online version for colours)

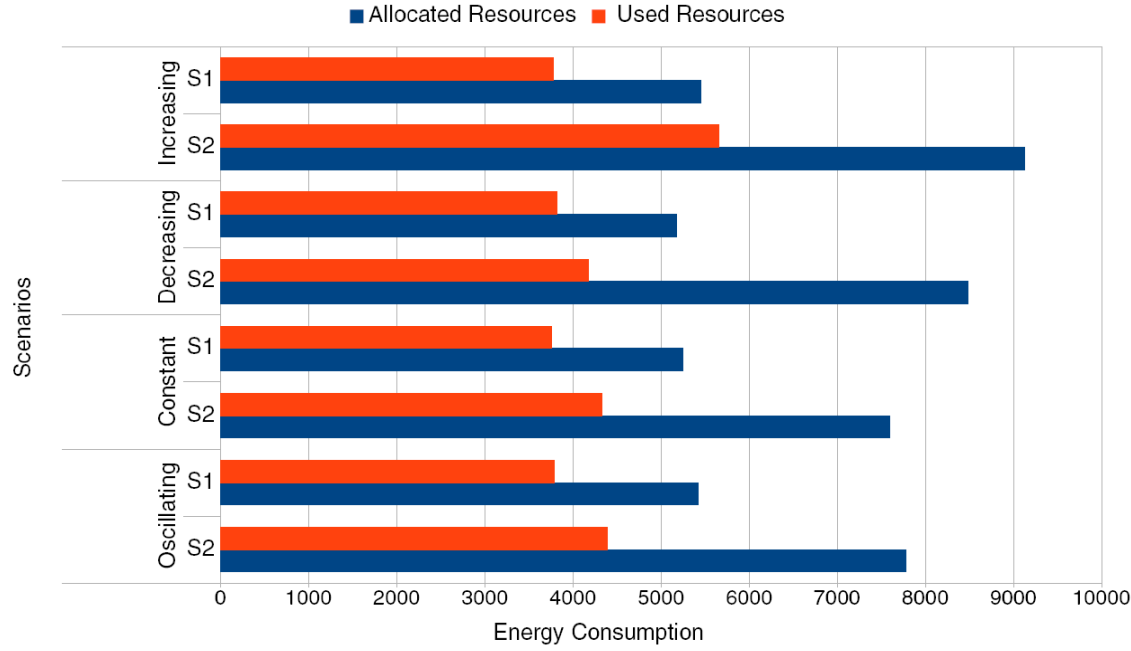

Figure 14 Resource consumption distribution in the different scenarios (see online version for colours)

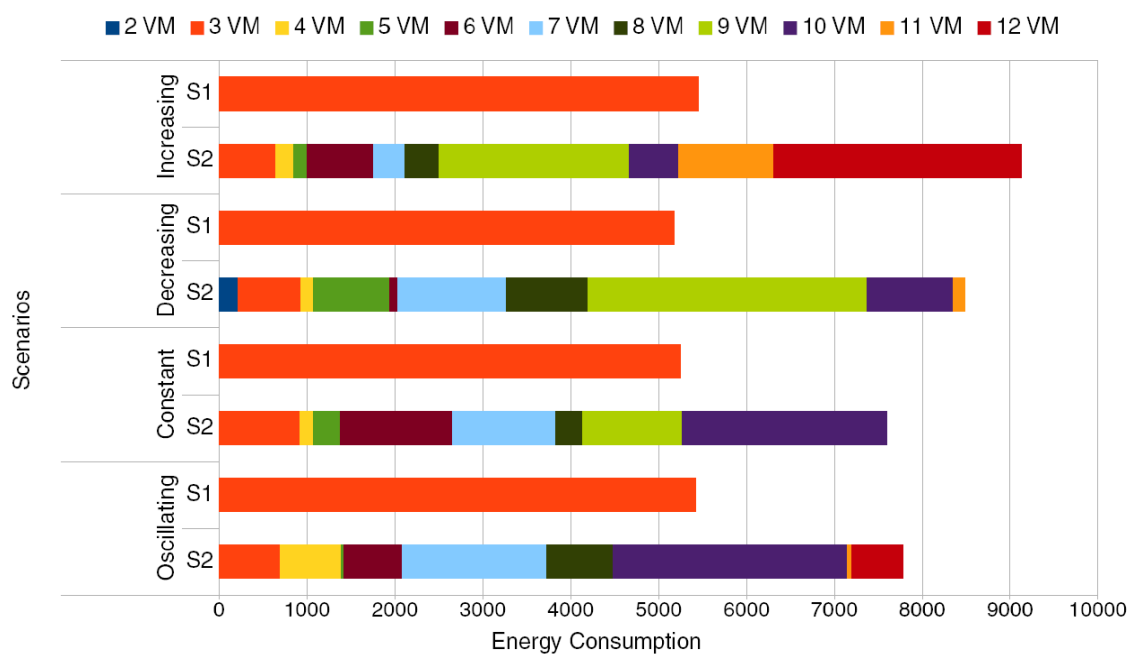


To calculate resource consumption, as we have the load in each monitoring observation, we use this value to calculate the percentage of resources that the application is occupying. For example, in a observations where there are $5 \mathrm{VMs}$ running and the calculated load is equal to $70 \%$, the resource consumption results in $3.5 \mathrm{VMs}(0.7 \times 5)$. So, we use this values to calculate the total resource consumption of all monitoring observations. In Figure 13 we compare resource consumption against resource allocation of each workload in two scenarios: (s1) application execution without using elasticity; and (s2) application execution using elasticity. Observing the figure, the workloads decreasing, constant and oscillating in scenario s2 achieved very close values of used resources (between 4,180 and 4,394 ) with a variation of $4.8 \%$. The increasing workload, in turn, achieved the highest value in the same scenario. It is important to emphasise that this was the workload that the application took more time to compute. Analysing resource consumption in scenario s1, all workloads achieved close values with variations up to $1.7 \%$. This reinforces that even though the workloads are different, the application computes the same portion of data for all workloads. As a result, when running the application without elasticity the resource consumption of all workloads tends to be the same.

Figure 14 depicts the profiles of VMs that compose the energy metric in scenarios s1 and s2. Each portion of the figure bars corresponds to the portion of energy that each set of VMs contributed in the final energy. The figure shows that lower values of energy were obtained by the executions with the smaller amounts of resources. In particular, the executions from scenario s1, where elasticity was disabled, used the lower possible amount of VMs (one per stage). On the other hand, the executions that used a greater number of VMs obtained the largest energy results. Regarding the impact of the elasticity, the executions of scenario s2 used at least $10 \mathrm{VMs}$. For instance, the Ascending workload in scenario s2 scaled up to $12 \mathrm{VMs}$ achieving the highest energy value. This behaviour shows that the greater the number of used VMs the higher the resource consumption.

\section{Conclusions}

This article presented Pipel, a model that provides automatic elasticity for pipeline applications. Pipel works at the PaaS level of a cloud, so users do not need to change their applications to take from the elasticity. With the aid of an elastic manager that monitors and rearranges the resources, it is possible to allocate or consolidate VMs according to the specific need of each stage of the application. Pipel introduces the concept of asynchronous multielasticity for pipeline applications, where we explore resource reorganisation at different stages always not blocking the normal execution of the application. Thus, scaling in and out operations happen in parallel with the application execution, being this last notified when the operation is completely done.
Pipel was evaluated through a prototype, which ran over two scenarios: with and without elasticity support. The results demonstrated that it is possible to achieve from $23 \%$ (worst case) to $38 \%$ (best case) of reduction on the application execution time with the use of elasticity. Despite the energy used during the execution of the application to allow the employment of elasticity, the cost metric (application time $\times$ energy consumption) says that the elasticity usage is not prohibitive when compared to a non-elastic execution. In brief, the results show that the larger the performance gains, the larger the energy consumption. This occurs because the application is CPUbound, so more processing elements tend to reduce the time but also enlarge the energy metric.

Future works include the development of an algorithm that evaluates the life of the VMs. Considering this parameter, we aim to reduce the costs of using the cloud, since most of the cloud providers charge for the entire hour, for instance, even if the resource is not used in the whole period. Moreover, today Pipel works with reactive elasticity, then we also plan to provide proactive elasticity to anticipate under-and over-loaded situations towards performing elasticity actions to address them beforehand.

\section{References}

Al-Haidari, F., Sqalli, M. and Salah, K. (2013) 'Impact of CPU utilization thresholds and scaling size on autoscaling cloud resources', IEEE 5th International Conference on Cloud Computing Technology and Science, December, pp.256-261.

Apostol, E., Baluta, I., Gorgoi, A. and Cristea, V. (2011) 'Efficient manager for virtualized resource provisioning in cloud systems', IEEE International Conference on Intelligent Computer Communication and Processing (ICCP), August, pp.511-517.

Beltran, M. (2015) 'Automatic provisioning of multi-tier applications in cloud computing environments', The Journal of Supercomputing, Vol. 71, No. 6, pp.2221-2250.

Bryant, R., Tumanov, A., Irzak, O., Scannell, A., Joshi, K., Hiltunen, M., Lagar-Cavilla, A. and de Lara, E. (2011) 'Kaleidoscope: cloud micro-elasticity via VM state coloring', Proceedings of the Sixth Conference on Computer Systems, EuroSys '11, ACM, New York, NY, USA, pp.273-286.

Cai, B., Xu, F., Ye, F. and Zhou, W. (2012) 'Research and application of migrating legacy systems to the private cloud platform with cloudstack', IEEE International Conference on Automation and Logistics (ICAL), August, pp.400-404.

Costa, F., de Oliveira, D., Ocala, K., Ogasawara, E., Dias, J. and Mattoso, M. (2012) 'Handling failures in parallel scientific workflows using clouds', SC Companion: High Performance Computing, Networking, Storage and Analysis (SCC), November, pp.129-139.

Dutta, S., Gera, S., Verma, A. and Viswanathan, B. (2012) 'Smartscale: automatic application scaling in enterprise clouds', IEEE 5th International Conference on Cloud Computing (Cloud), p.221228.

Galante, G. and de Bona, L.C.E. (2012) 'A survey on cloud computing elasticity', IEEE Fifth International Conference on Utility and Cloud Computing (UCC), November, pp.263-270. 
Guirado, F., Ripoll, A., Roig, C., Hernndez, A. and Luque, E. (2006) 'Exploiting throughput for pipeline execution in streaming image processing applications', Euro-Par 2006 Parallel Processing, pp.1095-1105.

Guoxin, L., Wanli, Z. and Lirong, W. (2015) 'Application of OPC to realize the communications between WINCC and master-slave PLC in the profibus network', IEEE Fifth International Conference on Big Data and Cloud Computing (BDCloud), August, pp.227-230.

Hartley, T.D.R., Fasih, A.R., Berdanier, C.A. and Ozguner, F. (2009) 'Investigating the use of gpu-accelerated nodes for sar image formation', IEEE International Conference on Cluster Computing and Workshops, pp.1-8.

Hendrix, V., Fox, J., Ghoshal, D. and Ramakrishnan, L. (2016) 'Tigres workflow library: supporting scientific pipelines on HPC systems', 16th IEEE/ACM International Symposium on Cluster, Cloud and Grid Computing (CCGrid), May, pp.146-155.

Herbst, N.R., Kounev, S. and Reussner, R. (2013) 'Elasticity in cloud computing: What it is, and what it is not', Presented as part of the 10th International Conference on Autonomic Computing, pp.23-27.

Imai, S., Chestna, T. and Varela, C.A. (2012) 'Elastic scalable cloud computing using application-level migration', IEEE Fifth International Conference on Utility and Cloud Computing (UCC), November, p.9198.

Islam, S., Lee, K., Fekete, A. and Liu, A. (2012) 'How a consumer can measure elasticity for cloud platforms', Proceedings of the 3rd ACM/SPEC International Conference on Performance Engineering, ICPE '12, ACM, New York, NY, USA, pp.85-96.

Jahn, J., Pagani, S., Kobbe, S., Chen, J-J. and Henkel, J. (2015) 'Runtime resource allocation for software pipelines', $A C M$ Trans. Parallel Comput., May, Vol. 2, No. 1, pp.5:1-5:23.

Kumar, V., Ravikumar, K., Aravinth, S.S. and Rajkumar, B. (2014) 'A message passing interface to support fast data access in distributed cloud environment along with master and slave communication', 2nd International Conference on Current Trends in Engineering and Technology (ICCTET), July, pp.309-312.

Li, J., Humphrey, M., Agarwa, D., van Ingen, C., Jackson, K. and Ryul, Y. (2010) 'Escience in the cloud: a modis satellite data reprojection and reduction pipeline in the windows azure platform', IEEE International Symposium on Parallel \& Distributed Processing (IPDPS), p.110.

Malawski, M., Juve, G., Deelman, E. and Nabrzyski, J. (2012) 'Cost-and deadline-constrained provisioning for scientific workflow ensembles in IaaS clouds', International Conference for High Performance Computing, Networking, Storage and Analysis (SC), November, pp.1-11.

Mao, M. and Humphrey, M. (2011) 'Auto-scaling to minimize cost and meet application deadlines in cloud workflows', $S C$ ' 11 Proceedings of 2011 International Conference for High Performance Computing, Networking, Storage and Analysis, No. 49.

Mattoso, M., Werner, C., Travassos, G.H., Braganholo, V., Ogasawara, E., de Oliveira, D., da Cruz, S.M.S., Martinho, W. and Murta, L. (2010) 'Towards supporting the life cycle of largescale scientific experiments', International Journal of Business Process Integration and Management, March, Vol. 5, No. 1, pp.79-92.
Orgerie, A-C., De Assuncao, M.D. and Lefevre, L. (2014) 'A survey on techniques for improving the energy efficiency of large-scale distributed systems', ACM Computing Surveys, March, Vol. 46, No. 4, pp.1-31.

Rajan, D., Canino, A. and Izaguirre, J.A. (2011) 'Converting a high performance application to an elastic cloud application', IEEE Third International Conference on Cloud Computing Technology and Science (CloudCom), December, pp.383-390.

Ramanath, R., Snyder, W.E., Yoo, Y. and Drew, M.S. (2005) 'Color image processing pipeline', IEEE Signal Processing Magazine, March, pp.34-43.

Righi, R., Rodrigues, V., da Costa, C.A., Galante, G., Bona, L. and Ferreto, T. (2016a) 'Autoelastic: automatic resource elasticity for high performance applications in the cloud', IEEE Transactions on Cloud Computing, April, Vol. 4, No. 1, pp.6-19

Righi, R.d.R., da Costa, C.A., Rodrigues, V.F. and Rostirolla, G. (2016b) 'Joint-analysis of performance and energy consumption when enabling cloud elasticity for synchronous HPC applications', Concurrency and Computation: Practice and Experience, cpe.3710, Vol. 28, No. 5, pp.1548-1571.

Rodriguez, M.A. and Buyya, R. (2015) 'A responsive knapsackbased algorithm for resource provisioning and scheduling of scientific workflows in clouds', International Conference on Parallel Processing, September.

Schneider, S., Andrade, H., Gedik, B. and Biem, A. (2009) 'Elastic scaling of data parallel operators in stream processing', IEEE International Symposium on Parallel \& Distributed Processing, IPDPS, May, pp.1-12.

Sharma, U., Shenoy, P., Sahu, S. and Shaikh, A. (2011) 'Kingfisher: cost-aware elasticity in the cloud. INFOCOM', Proceedings IEEE, April, pp.206-210.

Tran, G.S., Tchana, A., Hagimont, D. and de Palma, N. (2015) 'Cooperative resource management in a IaaS', IEEE 29th International Conference on Advanced Information Networking and Applications (AINA), March, p.611618.

Wen, X., Gu, G., Li, Q., Gao, Y. and Zhang, X. (2012) 'Comparison of open-source cloud management platforms: openstack and opennebula', 9th International Conference on Fuzzy Systems and Knowledge Discovery (FSKD), May, pp.2457-2461.

Wilkinson, B. and Allen, C.M. (2005) Parallel Programming: Techniques and Applications Using Networked Workstations and Parallel Computers. An Alan R. Apt Book, Pearson/Prentice Hall.

Wu, C-J., Ku, C-F., Ho, J-M. and Chen, M-S. (2016) 'A novel pipeline approach for efficient big data broadcasting', IEEE Transactions on Knowledge and Data Engineering, August, pp.17-28.

Zhang, X., Javaid, H., Shafique, M., Peddersen, J., Henkel, J. and Parameswaran, S. (2015) 'Oe-pipeline: elastic hardware/software pipelines on a many-core fabric', Design, Automation \& Test in Europe Conference \& Exhibition (DATE), March, pp.363-368.

Zhang, X., Shae, Z-Y., Zheng, S. and Jamjoom, H. (2012) 'Virtual machine migration in an over-committed cloud', Network Operations and Management Symposium (NOMS), April, pp.196-203. 